\title{
Breast sparganosis: a case report
}

\author{
Oh Chul Kwon ${ }^{1} \cdot$ Beom Seok Kwak ${ }^{1}$
}

Received: 19 October 2017/Accepted: 19 April 2018/Published online: 10 May 2018

(C) Indian Society for Parasitology 2018

\begin{abstract}
A 57-year-old Korean female patient presented with a migrated palpable left breast mass. Physical examination revealed a 3-cm soft, non-tender mass in the upper inner quadrant of her left breast. Mammography showed 8.2$\mathrm{cm}$ extended nodular and tortuous tubular masses in the upper portion of her left breast. Ultrasonography revealed a $5.8-\mathrm{cm}$ extended nodular, tortuous tubular and hypoechoic to anechoic lesion in the subcutaneous mammary parenchymal layer. A lumpectomy was conducted and revealed a $30-\mathrm{cm}$ live tapeworm. Histopathologic examination of the excised breast tissue revealed chronic granulomatous inflammation and eosinophilic infiltration by a parasitic organism, which was confirmed as a sparganum. After surgery, the patient was treated with a single dose of praziquantel and albendazole. Asian countries such as Korea, China and Japan are known to have high endemicity of sparganosis, mostly because of their dietary customs. When examining patients from these countries, sparganosis should be considered because of the unique dietary customs despite the high level of hygiene.
\end{abstract}

Keywords Breast - Sparganosis · Parasitic diseases . General surgery

\section{Introduction}

A 57-year-old Korean female patient presented with a migrated palpable left breast mass. She complained that the mass had been palpable for several months, and she

Beom Seok Kwak

kbs@dumc.or.kr

1 Department of Surgery, College of Medicine, Dongguk University, 27, Dongguk-ro, Ilsandong-gu, Goyang-si, Gyeonggi-do, Republic of Korea recognized that the mass had migrated from its original location recently. She denied experiencing systemic symptoms, including fevers or chills. She reported regular consumption of impure water from a mineral spring because of a superstitious belief about its therapeutic effects but denied consumption of frogs or snakes. Physical examination revealed a $3-\mathrm{cm}$ soft, non-tender mass in the upper inner quadrant of her left breast.

Mammography showed $8.2 \mathrm{~cm}$ extended nodular and tortuous tubular masses in the upper portion of her left breast, and disappearance of previously noted nodules on screening the mammography done 5 years ago (Fig. 1). Ultrasonography revealed a $5.8 \mathrm{~cm}$ extended nodular, tortuous tubular and hypoechoic to anechoic lesion in the subcutaneous mammary parenchymal layer. Vascularity increase was not observed (Fig. 2). The patient's routine laboratory test revealed no abnormality, including leukocytosis, neutrophilia, or eosinophilia. Meanwhile, the serum anti-sparganum IgG antibody level was 1.836 , which was positive (GENEDIA ${ }^{\circledR} \mathrm{Cy} / \mathrm{Sp}$ Ab ELISA, sensitivity $=100 \%$, specificity $=100 \%$, cut-off point $=0.229$ ).

A lumpectomy was conducted and revealed a $30 \mathrm{~cm}$ live tapeworm (Figs. 3, 4). Histopathologic examination of the excised breast tissue revealed chronic granulomatous inflammation and eosinophilic infiltration by a parasitic organism, which was confirmed as a sparganum.

After surgery, the patient was treated with a single dose of Praziquantel and Albendazole. There was no evidence of recurrence during 2 months of outpatient follow-ups.

The nodules shown in the mammography 5 years ago were $1 \mathrm{~cm}$ extended multiple indeterminate nodules in the upper outer portion of her left breast; it is uncertain if it was a parasite, because she didn't undergo further examination at that time. It was probably not a parasite, given the evidence from that mammography, because the 


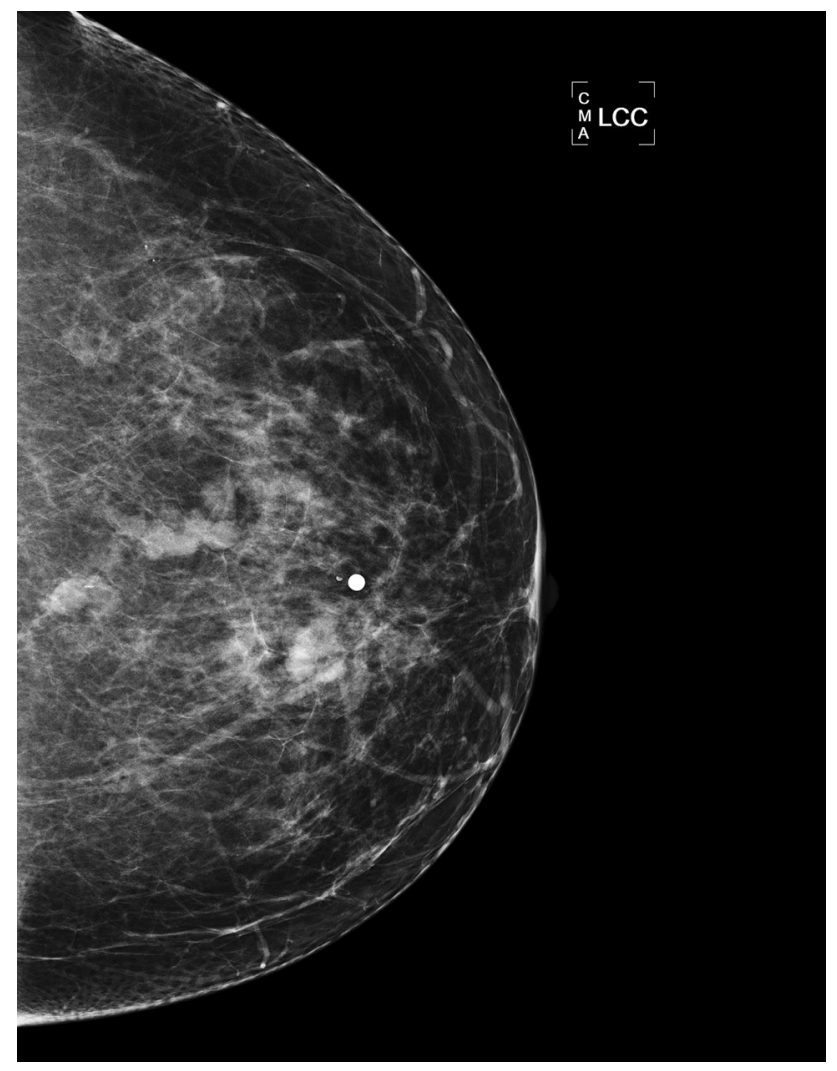

Fig. 1 Mammography showed an 8.2-cm extended nodular and tortous tubular masses on upper portion of left breast

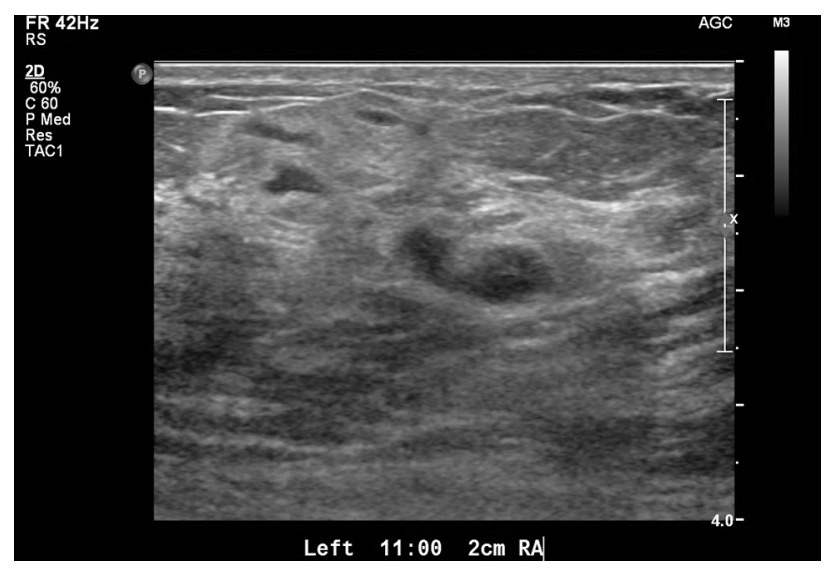

Fig. 2 Targeted ultrasound showed a nodular, tortous tubular hypoechoic lesion beneath the skin, measuring $5.8 \mathrm{~cm}$. There is an increased subcutaneous fat echogenicity without increased vascularity

morphological features are markedly different from those in other literature and present features (Song et al. 2015).

Asian countries such as Korea, China and Japan are known to have high endemicity of sparganosis, mostly because of their dietary customs (Lescano and Zunt 2013). In this case, Korea is a developed country, and safe drinking water is easily accessible. But many people drink

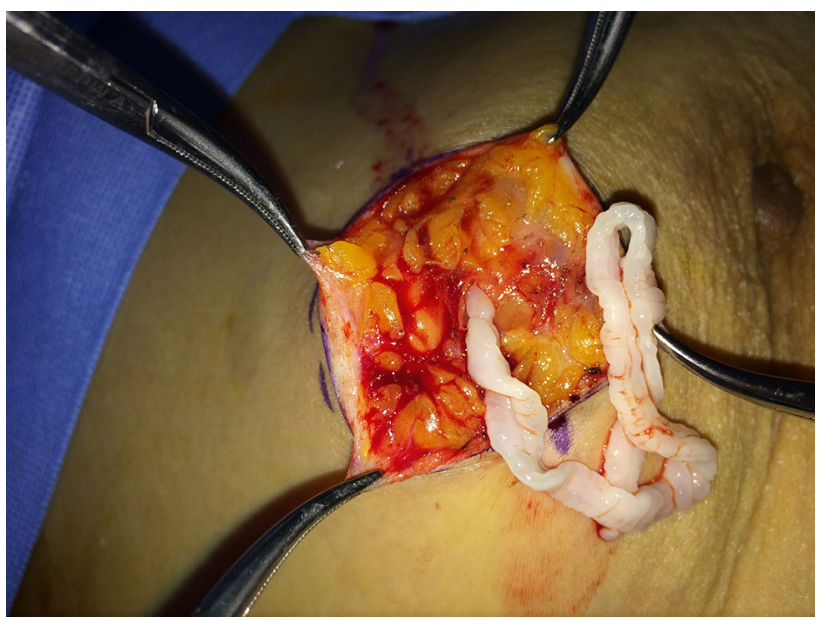

Fig. 3 Gross photograph of the tapeworm during surgery. The tapeworm was alive during surgery

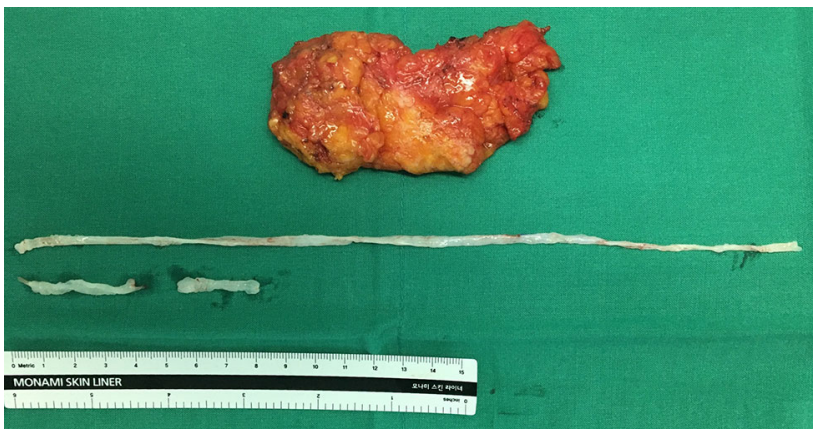

Fig. 4 Surgical specimen. The fragments of the 30-cm tapeworm below the excised breast mass

water from unsafe mineral springs for superstitious reasons, leading to high endemicity of sparganosis. Consequently, when examining patients from these countries, sparganosis should be considered because of the unique dietary customs despite the high level of hygiene.

\section{Compliance with ethical standards}

Conflict of interest The authors declare that they have no competing interests.

IRB statement This paper is a case report and does not require IRB approval as per the Institute's policy.

\section{References}

Lescano AG, Zunt J (2013) Other cestodes: sparganosis, coenurosis and Taenia crassiceps cysticercosis. Handb Clin Neurol 114:335-345

Song EJ, Sohn YM, Ryu KN, Min SY, Shin SH, Park YK (2015) Breast sparganosis and incidentally detected subcutaneous and intramuscular sparganosis at several sites: case report and literature review. Jpn J Radiol 33:225-228 